\title{
Next-generation sequencing: recent applications to the analysis of colorectal cancer
}

\author{
Filippo Del Vecchio', Valentina Mastroiaco ${ }^{2}$, Antinisca Di Marco², Chiara Compagnoni², Daria Capece ${ }^{3}$, \\ Francesca Zazzeroni ${ }^{2}$, Carlo Capalbo ${ }^{4}$, Edoardo Alesse $^{2}$ and Alessandra Tessitore ${ }^{2^{*}}$ (1)
}

\begin{abstract}
Since the establishment of the Sanger sequencing method, scientists around the world focused their efforts to progress in the field to produce the utmost technology. The introduction of next-generation sequencing (NGS) represents a revolutionary step and promises to lead to massive improvements in our understanding on the role of nucleic acids functions. Cancer research began to use this innovative and highly performing method, and interesting results started to appear in colorectal cancer (CRC) analysis. Several studies produced high-quality data in terms of mutation discovery, especially about actionable or less frequently mutated genes, epigenetics, transcriptomics. Analysis of results is unveiling relevant perspectives aiding to evaluate the response to therapies. Novel evidences have been presented also in other directions such as gut microbiota or CRC circulating tumor cells. However, despite its unquestioned potential, NGS poses some issues calling for additional studies. This review intends to offer a view of the state of the art of NGS applications to CRC through examination of the most important technologies and discussion of recent published results.
\end{abstract}

Keywords: Next-generation sequencing, Colorectal cancer, Precision medicine, Targeted therapy

\section{Background}

Since the application of modern technology in medicine, scientists always tried to understand the real nature of nucleic acids. To this end, a great innovation was brought by the pioneer work of Sanger et al. in the late 70s, when they elaborated the most used method to sequence DNA [1], still considered as the gold standard in molecular diagnostics, even though it is expensive and time-consuming. Growing efforts have been made to widen technical knowledge in this field until the discovery of the "second and third-generation sequencing" methods [2, 3]. They are both part of the next-generation sequencing (NGS) technology, a group of techniques revolutionizing the standard concept of nucleic acids sequencing. The great success of NGS technology is due to the capability of massively sequencing millions of DNA reads, with the possibility to perform, at least, multi-gene analysis, by

\footnotetext{
*Correspondence: alessandra.tessitore@univaq.it

${ }^{2}$ Department of Biotechnological and Applied Clinical Sciences,

University of L'Aquila, L'Aquila 67100, Italy

Full list of author information is available at the end of the article
}

using very low amount of nucleic acids. NGS technology is suitable for rapid and efficient sequencing of complex genomes too, with consequent time and cost reduction. Furthermore, it can also count on a noteworthy flexibility: in fact, its application has been reported to be successful in different research fields such as molecular diagnostics of genetic diseases, infectious diseases, cancer and pharmacogenomics [4-7].

Many studies on cancer took advantage of the use of this technology, due to the possibility to detect high numbers of variants, related to complicated mechanisms of oncogenesis and tumor heterogeneity [8,9]. Today, molecular profiling of tumors can provide information about diagnosis, prognosis, prediction to therapy response, which can drive clinical decision making. Concerning the precision care in cancer, the eligibility for targeted drugs, able to specifically inhibit aberrantly dysregulated mechanisms, is strictly affected by the "mutational status" of specific genes. The adoption of this type of medication refers to FDA-approved drugs for a specific tumor type, off-label therapies for specific molecular 
lesions in a non-approved tumor type or to clinical trials focused on analyzing the effects of agents based on the presence of well-defined molecular alterations. In this context, deep molecular characterization of tumors is a key element for optimal patient's management and unified guidelines to detect and classify variants, interpret and report results should be recommended and adopted [10].

Colorectal cancer (CRC) is one of the most interesting fields of NGS application. CRC is among the most lethal and frequent types of tumors in the industrialized world and is characterized by a great quantity of activating mutations [11]. These features are the reason why the number of studies employing next-gen techniques is rising in the last years. Their use led to the production of significant results about the identification of novel mutations/altered genes or genomic rearrangements and the possible evaluation of therapy response. This review is intended to condense the state of the art about next-gen technologies and their applications in CRC throughout the last years to provide a useful synopsis. We describe and discuss: (i) NGS main applications and platforms, (ii) main features of CRC pathogenesis and therapy, (iii) NGS application in CRC, by taking into consideration diagnostics of actionable genes, novel mutations, less frequently mutant genes, transcriptomics, epigenetics alterations, other possible directions. Finally, we present some concluding remarks.

\section{NGS technology \\ NGS applications}

Next generation sequencing technologies are increasingly used in many fields. Their power consists in the possibility to obtain huge amount of data and discover novel and essential information about the human genome. This feature opened many contexts of successful applications. The first was whole-genome sequencing (WGS), an approach intended for entire genome sequencing. It provides the most complete landscape of genomic information and possible biological consequences [12]. Despite its potential, which permits the discovery of undescribed mutations at the level of coding as well as non-coding regions, mostly involved in the regulation of gene expression, it shows undeniable difficulties due to the high amount of generated data and their validation (i.e. variants with unknown significance-VUS-, intronic mutations, etc.). Furthermore, the method requires considerable human resources for efficient organization and interpretation [13]. To avoid these difficulties and obtain at the same time extensive high-throughput data, less labored methods, such as whole-exome sequencing (WES), targeted sequencing [14] or transcriptome sequencing (RNA-Seq), have been conceived. The first one provides only information about exons, restricting the length of the nucleic acid analyzed within coding regions; whereas targeted sequencing is focused on specific subsets of regions, or more relevant genes, whose pathogenic involvement in specific diseases has already been described or supposed. Transcriptome analysis generates data about splicing variants, allelic expression [15], RNA editing [16] and alternative 3'-UTR polyadenylation as well [17]. Finally, the study of epigenetic modifications is becoming an emerging field of NGS application, particularly in cancer research. The analysis of elements playing a role in such mechanisms, such as methylated sequences, DNA-binding proteins or non-coding RNAs, could aid in defining profiles that can be used for diagnostic and/or prognostic purposes [13, 18].

\section{Next-generation platforms}

Automated Sanger sequencing is still considered as the "gold standard" in molecular diagnostics. As above mentioned, cancer is a very complex disease, characterized by abnormal functions of multiple genes and sophisticated mechanisms physiologically directed to protect normal cell functions. The entry of new generation technologies in cancer research provided the possibility to perform multi-gene analysis, contributing to shed light on complicated molecular mechanisms of oncogenesis and, more, offering a great contribution at the translational level in the field of the precision medicine. Big companies have developed high-performing instruments able to massively generate hundreds of thousands of sequencing reactions in parallel. These technologies reach good standards of quality and reliability, and are now used in an increasing number of laboratories worldwide for multi-gene or even genome, exome and transcriptome analysis. Basically, they work by using dye terminators, pyrosequencing, monitoring $\mathrm{pH}$ changes or sequencing at single-molecule level. Recently, nanopore-based technologies have been applied as well. A brief description of the most common platforms is provided below, along with their main features.

\section{Roche}

The first NGS technology was released in 2005 with the 454 Genome Sequencer (Life Sciences, today Roche). The great initial success can be attributed to its key point: the association between emulsion PCR, a new amplification strategy, and pyrosequencing. Emulsion PCR is an innovative methodology which uses small water droplets scattered in a lipid solution, where individual DNA fragments are amplified. Briefly, DNA is fragmented, ligated to adapters and mixed to micro-beads containing complementary adapters. DNA fragments-beads complexes are emulsified in droplets containing PCR reactants, so 
that each droplet contains a single copy of DNA fragment (or a single allele) to be amplified. Afterwards, a standard PCR reaction amplifies DNA. At the end of the procedure, every bead carries on its surface even hundreds of thousands of amplified fragments. Samples are then loaded onto the wells of a picotiter plate to perform many thousands of pyrosequencing $(\mathrm{PPi})$ reactions in parallel by sequentially adding, one at a time, the four deoxynucleotides. A CCD camera detects light signals. Due to the PPi chemical/physical features, this technology can give rise to errors within homopolymeric stretches, with consequent mistakes in the estimated length and introduction of "indel" errors. The two most recent platforms, GS Junior and GS FLX, greatly improved the output, with significant read lengths of 400 and 1000 nucleotides (nt) respectively, close to that reached by Sanger single-gene sequencing, and maximum throughput performance around $700 \mathrm{Mb}$ (Table 1) [19].

\section{Illumina-solexa}

Genome Analyzer, the first Solexa sequencer, was launched in 2006, giving scientists the possibility to analyze $1 \mathrm{~Gb}$ of data in a single run. In 2007, the company was acquired by Illumina. The Illumina sequencing technology is based on the use of clonal arrays coupled to clonal massive sequencing by synthesis (SBS) by using cyclic reversible termination (CRT). In brief, after library preparation, DNA fragments, ligated to specific adapters, are provided. Library fragments hybridize to oligos immobilized onto a flow cell and polymerization of a complementary strand occurs. After, the template is washed away and the immobilized complementary single-stranded fragment of new synthesis is in situ amplified by the original mechanism of bridge amplification. This process is repeated to produce billions of clusters which result in clonal amplification of all the fragments. Then sequencing takes place by fluorescently-labeled nucleotides' incorporation, detected by light source excitation. Identical fragments are massively sequenced in parallel, basecalling is determined by emission wavelength and signal intensity as well. The recent 2-channel technology, which uses a mix, instead of 4 , specific dyes, further improved the process, by maintaining the same level of accuracy and reducing the number of image acquisitions per cycle from 4 to 2 , with consequent time saving. The complete procedure is directed to sequence forward and reverse strands: therefore, the final analysis considers data from both strands. The applications of Illumina instruments range from genomics to transcriptomics and epigenomics. To meet different needs, the company offers a series of several versatile instruments characterized by different levels of performance (MiniSeq, MiSeq, NextSeq, HiSeq and HiSeq X). The read length ranges from 150 to $300 \mathrm{bp}$, with more than $99 \%$ accuracy (Table 1) [20].

\section{Ion Torrent Thermo Fischer}

Ion Torrent technology is based on original chemical/ physical principles, different from those characterizing the above-mentioned next-generation platforms. Commercialized in 2010 by Life Technologies, it is a semiconductor-based technology where minimal $\mathrm{pH}$ changes, produced by the release of hydrogen ions as by-product of nucleotide incorporation, are detected. This is possible by using an "Ion chip", structured into two parts, to deliver reactants and communicate directly with a proton detector for nucleotide identification during the reaction of incorporation. Different from the other NGS technologies, Ion Torrent recognizes added nucleotides avoiding the use of fluorescence. In fact, the instrument interrogates one nucleotide at a time and incorporation's specificity is guaranteed by the release and detection of $\mathrm{H}^{+}$ ions. In this case, the most frequent errors are caused by phasing. This means that, especially for homopolymer sequences, not all DNA fragments could incorporate nucleotides at each step. Nonetheless, the error rate for this system is very low (i.e. 1\%). Continuous improvements have increased read length from the initial 100 to $200 \mathrm{nt}$ (actual average value). Ion Torrent throughput made an even greater jump, starting from $10 \mathrm{Mb}$ to the current maximum of $15 \mathrm{~Gb}$ (Table 1) [21].

\section{SOLiD}

SOLiD are the initials of sequencing by hybridizationligation implemented in oligonucleotide ligation and detection. This technology was originally developed by Applied Biosystems. The system shares with 454 Roche the emulsion PCR as first amplification step during the DNA library construction. But the very distinctive feature of SOLiD platforms is in the chemistry of its sequencing phases. Amplified fragments are placed on surfaces and subjected to multiple hybridization and ligation reactions according to a fluorescent dye scheme. Each dye labels four dinucleotides for a total of 16 dinucleotides combinations labeled by four different fluorescent dyes. In this way each position is labeled twice, therefore, after two sequential ligation events, the resulting color will identify the incorporated nucleotide. This system is also useful to distinguish incorrect sequence identification from sequence polymorphism. Indeed, the former is detected only in one of the two ligation reactions whereas the latter is detected in both reactions [21,22].

\section{Pacific Biosciences}

In 2010, Pacific Biosciences Inc. launched into the market its own innovative platform PacBio, based on the 


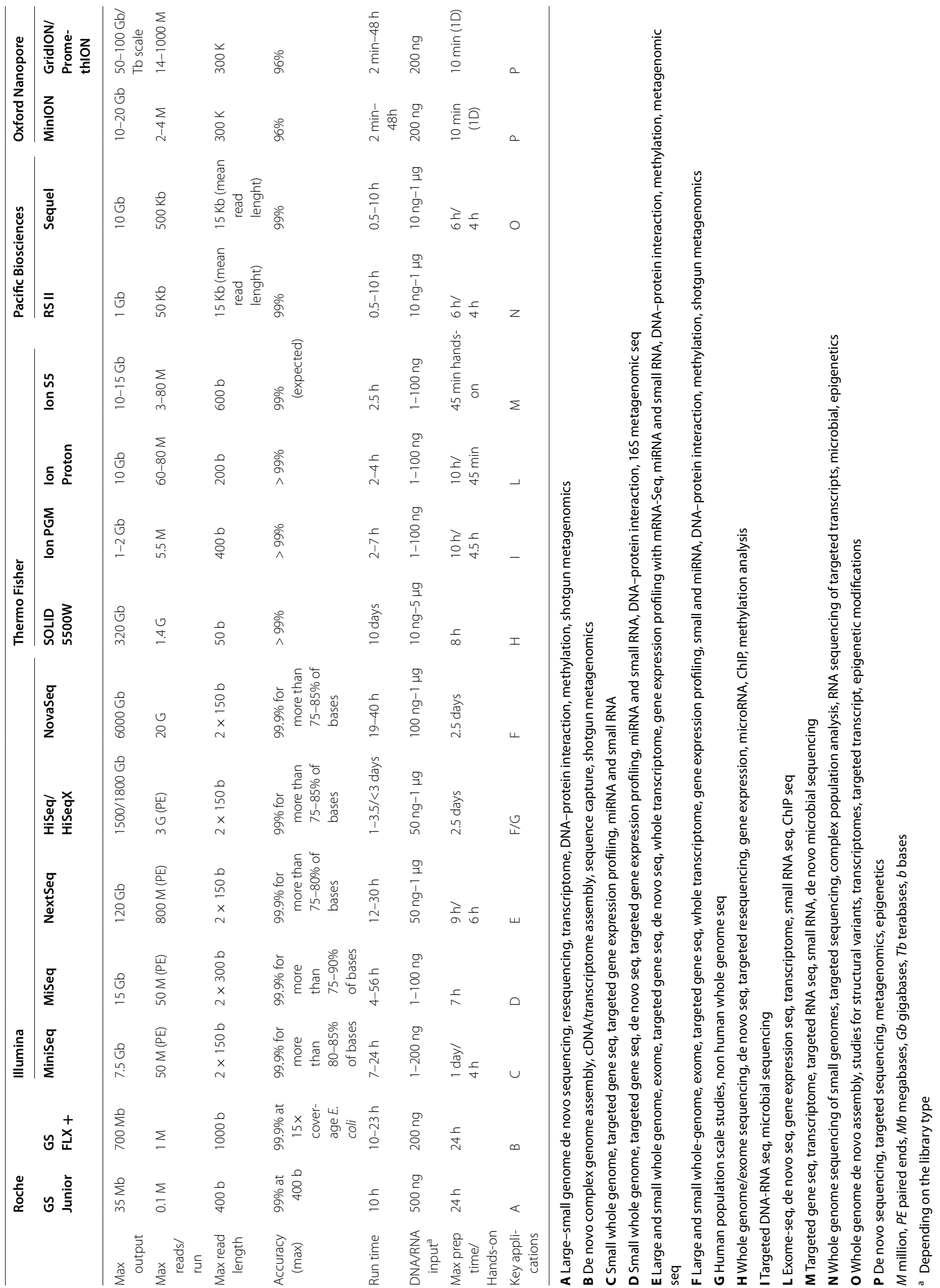


new approach of single-molecule real-time sequencing (SMRT) [23]. Ten of thousands of Zero-Mode Waveguides (ZMWs) chambers, with the smallest light detection volume, in SMRT cells are illuminated from below at the bottom, where DNA polymerase and template are immobilized. After addition of specifically labelled phospho-linked nucleotides, the system detects the incorporation of each of them, revealed by specific fluorescent light emission. The process occurs simultaneously in thousands of ZMWs, providing millions of sequencing reads. Due to these features, the system is exposed to errors caused by missing registration of base incorporation or by wrong interpretation of nucleotide dwelling in the active sites. However, the current error rate is only $0.1 \%$. Concerning read length, PacBio platforms show great performance (up to $20,000 \mathrm{bp}$ read length) up to $10 \mathrm{~Gb}$ (Table 1) [24].

\section{Oxford Nanopore technologies}

In 2012 Oxford Nanopore announced a new technology able to directly sequence a DNA fragment by measuring the change in current flow, due to the passage of such molecule through a nanopore embedded within a membrane $[25,26]$. MinION, the main platform today available, is a portable, small (100 g weight), USB powered apparatus provided by a flow cell with 2048 individually addressable nanopores which are controlled, in four groups of 512 , by application-specific integrated circuit (ASIC). Briefly, ends of 8-10 Kb genomic/cDNA fragments are ligated to different adapters identified as lead, hairpin and training. Lead adapter promotes capture and loading of a processive enzyme at the $5^{\prime}$ end of a single strand. DNA molecule is captured at the level of an empty nanopore (open channel) and the enzyme activates the strand's translocation through the pore, ensuring unidirectional single-nucleotide shift. After the hairpin adapter, linking strands each-other, passes through the enzyme, the same process takes place for the complement strand. The passage of trailing adapter leaves the nanopore empty (open channel). As the molecule moves through the nanopore, sensors in the detection system detect changes in ionic current, due to differences in the nucleotide sequence. The current changes are computationally elaborated as a sequence of 3-6 nucleotide long kmers ("words") using graphical models. The maximum read length is now approaching $1 \mathrm{Mb}$, with base calling accuracy up to 99\%, the time to first usable read is $2 \mathrm{~min}$. GridION X5 and PromethION are more processive platforms which combine multiple MinION devices (Table 1) [27].

\section{Qiagen GeneReader}

Very recently, Qiagen launched into the market the GeneReader system [28]. This is a standardized NGS workflow designed from nucleic acids' extraction to insight and specifically directed on analysis of cancer genes' panel. For this purpose, the QCI Qiagen clinical insight is provided to interpret data and to quickly identify genetic markers associated with approved therapies. The GeneReader platform is based on the SBS technology and is characterized by high scalability and flexible throughput. The system was successfully validated on FFPE CRC samples in comparison to other known platforms [29].

\section{Bionano technologies}

Bionano genomics put sequencing technologies on new track using the optical mapping concept. Its great innovation is based on the possibility to fluorescently label sequence-specific traits of long, high-molecular weight DNA (up to $1 \mathrm{Mb}$ ) to have an optical barcode per each DNA molecule. DNA is then loaded in nanotunnels and channels where it is linearized and imaged by a highresolution camera. The images are converted into digital label patterns which are de novo assembled by dedicated algorithms to fully reproduce the original genomic map [30]. Due to these features, Bionano genomic mapping technologies allow to increase detection rates of large structural variations and improve assembly contiguity which can be missed because of the too small and fragmented reads generated by conventional NGS sequencing. A recent study confirmed the great potential of this technology in the discovery of novel genetic rearrangements in cancer [31].

\section{NGS platforms comparison}

The possibility to use multiple NGS platforms gave rise to comparisons aimed to evaluate the different characteristics among them. This could help in achieving data to define singular key aspects of the platforms and provide directions about their outputs and when they can better perform. A comparison among the most relevant technical features of most of the instruments previously described is reported in Table 1 , where differences are identified in terms of maximum output, reads per run and read length, accuracy, run time, amount of nucleic acids necessary for analysis, experimental time, and key applications. NGS platforms can generate short (SOLID, Ion Torrent, Roche, Illumina) or longer reads (PacBio, Oxford) [32]. Considering the main differences among platforms belonging to each group, Roche and Ion Torrent display higher read lengths with respect to Illumina, but, on the other hand, produce more indel errors, especially within homopolymer regions [33, 34]. Ion Torrent is fast and versatile, making it possible, depending on the necessity, to use different types of chips with specific related yield [21]. Conversely, Illumina is less 
predisposed to homopolymer errors, it shows an overall accuracy greater than $99.5 \%$, but sometimes can provide under-representation of regions (i.e. AT/GC-rich) and nucleotide substitution errors [35-38]. Both PacBio and MiniONS platforms generate much longer reads and are more suitable for de novo genome assembly or transcript sequencing. PacBio shows high error rate (10-15\%), consisting in common indel errors [39], but fortunately they are casually reported within each single sequencing iteration (single-pass). For this reason, the problem may be overcome by using an adequate coverage [40]. MinION is an easily portable device, USB provided, which shows some limitations in the analysis of very long fragments [32]. Due to the technology, providing huge number of distinct signals, it displays large error rate, mainly indels. In addition, homopolymers cannot be accurately sequenced, being difficult precisely distinguish in the nanopore signals due to the same type of "leaving" and "entering" nucleotide [32]. The same limitation appears with modified nucleotides, altering the typical nucleotide-dependent voltage variation.

Several studies have been addressed to compare NGS platforms' overall performance in various fields. Roche 454, Ion Torrent PGM and MiSeq were in parallel used in metagenomics [41] and differences were highlighted: Ion Torrent resulted unbeatable for speed, Roche 454 produced longer reads, whereas MiSeq provided greater coverage depth and breadth. Quail et al. [42] evaluated the performance of Ion Torrent, PaciBio and Illumina to sequence 4 microbial genomes with CG content ranging from 19.3 to $67.7 \%$. They showed that Ion Torrent detects more variants, but also gives more false-positive results. Moreover, context-specific errors were detected in PGM and MiSeq, but not in PacBio instruments. Another work [43] described the use of Ion Torrent and Illumina HiSeq 2000 for the analysis of a Rhodobacter sample with high GC content. Ion Torrent sequencing quality was more stable than HiSeq 2000, where decay of fluorescence signal occurred, and superior in terms of GC depth distribution reproduction. Other published data display higher sensitivity of PacBio RSII technology compared to classical PGM and MiSeq, with identification of mutations in stool DNA at $0.5 \%$ frequency [44]. Some papers focused the attention on the strength of different platforms in cancer somatic mutation detection. Misyura et al. [45] compared the performance of MiSeq and Ion Proton by analyzing FFPE samples with amplicon-based commercial panels, consisting of approximately 50 relevant genes in cancer pathogenesis (MiSeq-APC and Proton-CHP). They evidenced $100 \%$ concordance in genomic regions subjected to analysis by both panels, including 27 low-frequency $(<15 \%)$ variants. Ion Proton, contrary to MiSeq, resulted suitable also for the study of low quality/ quantity DNA. This NGS dual approach, characterized by different chemistries, allowed to accurately identify even low-frequency somatic mutations, not detectable by conventional Sanger sequencing. Another group [46] used HiSeq or NextSeq to analyze NGS panels spanning 47 genes (relevant in pheochromocytomas, breast, CRC, renal, pancreatic and ovarian/uterine cancers) in 20,000 hereditary cancers. In this study, almost 8000 non-polymorphic variants were detected and further subjected to validation by Sanger sequencing. Among them, approximately $1.3 \%$ of NGS results, mainly located in complex genomic regions (i.e. A/T, C/G rich, homopolymer), were identified by both platforms as false positive. Conversely, simulating zero false-positive rate, the sensitivity of the assay decreased from 100 to $97.8 \%$, with 176 (2.2\%) clinically relevant variants detected by Sanger sequencing and missed in NGS. These results, obtained just after processing a huge number of samples, indicated that analysis parameters and threshold levels should be appropriately set-up by a bioinformatics pipeline. In addition, Sanger sequencing should be used to confirm NGS results as well. A comparison between GS-454 and Ion Torrent was provided by Hinrichs et al. [47]. This study was focused on analyzing the most used methods for detecting KRAS/ EGFR mutations in 25 FFPE lung cancer samples, already genotyped by Sanger sequencing. Ion Torrent technology (Ampliseq cancer panel) performed better than GS-454 (5 amplicons covering KRAS/EGFR hot spot) which failed in identifying KRAS mutations in four samples.

\section{Colorectal cancer (CRC) \\ CRC: main features}

Colorectal cancer (CRC) is referred to tumors affecting colon and rectum and represents the third most common type of tumor worldwide [11]. Although it is included among the worst malignancies, its incidence in the Western countries, particularly in high-income nations, remained almost constant during the last 20 years, whereas it appeared increasing in Eastern countries (Eastern Europe included). CRC are infrequent under 45, being $70 \%$ of patients diagnosed over 65 years [48]. Many important progresses have been made for therapeutic procedures, paying attention to the optimization of surgical resection. Diet and physical exercise are two central points in CRC prevention. Studies evidenced that red meat, alcohol and smoke abuse as well as obesity are important risk factors; on the contrary, physical activity seems to protect against the tumor $[49,50]$. Additional recognized risk factors are the so-called "inflammatory bowel diseases": in fact, several studies stated that the presence of an ulcerative colitis or Crohn's disease implied a greater risk of CRC occurrence [51, 52]. As for other cancers, patients who have been affected by a 
previous CRC are at risk for developing a second tumor [53].

\section{Molecular genetics of CRC}

Colorectal cancer is a very heterogeneous type of cancer and accounts for either sporadic or hereditary form. Sporadic tumors are more frequent compared to the inherited, which correspond to only $5 \%$ of cases [54]. The latter are defined as familial adenomatous polyposis (FAP) [55], hereditary non-polyposis colorectal cancer (HNPCC) [56] and MUTYH associated polyposis (MAP) [57], characterized by the presence of APC, MMR and MYH gene lesions, respectively. CRC was the first type of tumor described in its progression, serving as a model for the characterization of solid tumors' carcinogenesis. In '90s, Fearson and Vogelstein developed the "adenoma-carcinoma sequence" [58], concluding that the progression from an adenomatous polyp to invasive carcinoma is due to sequential acquisition of somatic mutations in different genes (i.e. APC, KRAS, BRAF, SMAD4, TP53). They described deletions on chromosome $5 \mathrm{q}$ regions, linked to the adenomatous polyposis coli (APC) gene, and subsequent activating mutations of KRAS gene as associated with the early phases of carcinogenesis. Additional deletions in the adenoma-carcinoma sequence were reported on chromosome 18q, related to deleted in colon cancer (DCC) gene. Then, other deletions at the level of chromosome 17p were detected, with tardive mutations affecting TP53. The general acceptance of this theory by the scientific community led to define colorectal cancer carcinogenesis as a process characterized by multiple sequential mutations.

Genetic instability was considered a leader event in CRC as well, and distinct pathways correlated to the disease have been identified. Chromosomal instability (CIN) accounts for $70-85 \%$ of cases and is characterized by accumulation of numerical and structural chromosomal abnormalities or loss of heterozygosity (LOH). This pathway is associated to lesions at the level of several genes, such as APC, KRAS or TP53 genes [59, 60]. Microsatellite instability (MSI) is defined as a hypermutable phenotype, causing a great number of genetic errors, due to mutations affecting genes acting in the repair of the mismatch (MMR) [61]. In addition, dysregulated epigenetic mechanisms have been described [62]: tumors with a $\mathrm{CpG}$ island methylator phenotype (CIMP) were reported even in early lesions of the colonic mucosa [63]. Furthermore, it is known that approximately $15 \%$ of CRC, mostly sporadic, show also MSI, arising from the MLH1 promoter methylation [64].

\section{Connections between CRC and gut microbiota}

Recently, the existence of an influence exerted by gut microbiota on CRC development has been highlighted, since gut microbiota seems to be directly involved in the regulation of intestinal immune system and promotion of intestinal inflammation. Generally, in healthy conditions, main components of the gut microbiota are represented by obligate aerobic bacteria. When this situation is altered, there is a change in microbiota composition and aerobic bacteria become to be replaced by facultative anaerobic ones. This condition, called "dysbiosis", is dangerous because intestinal tissue begins to be populated by microbes able to induce inflammatory processes [65]. The creation of an inflamed microenvironment predisposes to carcinogenesis. In this case, a study on mice [66] demonstrated that the integrity of the intestinal barrier is deteriorated with facilitated invasion of tissue by microbes and massive production of cytokines maintaining the inflammatory state. Proinflammatory interleukins, such as IL-17 and IL-23, are released and sustain the tumor growth. Inflammation caused by dysbiosis could also stimulate carcinogenesis through the selection of the most tumorigenic bacterial strains [67]. DNA damage resulting from the production of pro-oxidative molecules, such as reactive oxygen (ROS) and nitrogen (NOS) species is linked to bacteria as well $[68,69]$. Even if relatively young, the field of gut microbiota-CRC interactions is under growing investigation, due to the increasing number of reports evidencing connections with CRC tumorigenesis. Further analyses will add this new perspective to the CRC induction mechanisms.

\section{CRC therapy}

The classical way to treat CRC is surgical resection followed by chemotherapy. It is known that approximately one-fourth of CRC diagnosed patients show synchronous metastasis and almost a half develop metastasis after diagnosis [70]. During last years, drugs able to specifically target dysregulated molecules, with consequent prognosis amelioration, can be used in well-defined cases in association to cytotoxic treatment: this is the case of monoclonal antibodies against VEGF (Bevacizumab) and EGFR (Cetuximab and Panitumumab). No biomarkers are in use for anti-VEGF, whereas predictive biomarkers have been approved and now extensively considered for anti-EGFR therapy in clinical practice [71]. Many studies demonstrated the importance of KRAS and the closely related NRAS genotype in considering the response to anti-EGFR drugs, whose efficacy is mostly observed in KRAS and NRAS wild-type (WT) patients. On the contrary, patients with KRAS or NRAS mutations at the level of exons 2, 3 or 4 do not benefit of anti-EGFR treatment, which can even show detrimental effect when associated to oxaliplatin [72, 73]. However, it is known that a percentage of KRAS or NRAS WT patients is not responsive to EGFR targeted therapy, leaving to hypothesize that 
additional mediators could be involved in the dysregulation of molecular mechanisms leading to tumor initiation and development. Among them, BRAF and PI3KCA, acting downstream of RAS and involved in the PI3K/Akt/ mTOR signaling pathway, have been taken into consideration and are under investigation. With this regard, the 2016 ESMO guidelines recommend BRAF genotyping in KRAS/NRAS WT patients. On the other hand, mutations at the level of PI3KCA seem to predict resistance to anti-EGFR and for this reason the protein is considered as a target for inhibition in clinical trials in progress. Seen in this context, additional potentially actionable genes cannot be excluded. Due to its feature, NGS provides a suitable, fast and cost-effective technology to simultaneously identify multiple genes carrying either described or undescribed mutations which could play an active role in tumor development and, possibly, in driving therapeutic decision making.

\section{NGS in CRC}

\section{Mutation analysis of actionable genes}

Next-generation sequencing provides a fast high-throughput and cost-effective technology with respect to traditional Sanger sequencing to accurately identify mutations in known genes and to provide information of clinical utility [74]. Here we report the results of some of the most recent studies, summarized in Table 2. Peeters et al. [75] investigated the response to panitumumab in patients affected by metastatic CRC (mCRC). Adopting a massive multigene NGS sequencing (Roche GS FLX), the authors analyzed 9 genes in 320 samples and detected mutations in K/NRAS, BRAF, PI3KCA, PTEN, TP53, EGFR, AKT, CTNNB1 genes with frequencies ranging from 60 (TP53) to $1 \%$ (AKT). They demonstrated that panitumumab treatment prolonged progression free survival (PFS) in KRAS-WT patients compared to KRAS-mutant patients. Moreover, patients with KRAS- and NRAS/ BRAF WT genes showed better response with respect to KRAS-WT and NRAS/BRAF mutant patients. In conclusion, the study provided evidence that NGS can be a suitable method to identify predictive biomarkers. A work by Kothari et al. [76] on 468 CRCs, 77 of them already analyzed by standard test for KRAS in approved laboratories, demonstrated the viability and even the higher diagnostic power of Illumina NGS with respect to standard methods in identifying KRAS mutations, making it possible the detection of lesions with potential clinical impact, not typically evidenced by standard tests. A study by Ciardiello et al. [77] was focused on the analysis of twenty-two cancer-related genes in 182 KRAS exon 2 WT tumor samples from mCRC patients treated with first-line FOLFIRI plus cetuximab. Semiconductor-based NGS revealed one or more (up to 5) gene mutations in
124 out of 182 specimens. KRAS exon 2 mutations were detected in approximately 16\% (29/182) of patients, previously classified as wild-type by local laboratory tests, and TP53, KRAS, NRAS, PI3KCA and BRAF were described as more frequently mutated genes as well. Based on NGS gene mutation analysis results, evaluation of ORR and PFS provided information about a group of patients (KRAS and NRAS WT) which could benefit of the FOLFIRI plus cetuximab treatment, confirming the possible NGS use in clinical practice. Bai et al. [78] further highlighted the clinical utility of targeted gene sequencing. They analyzed 91 rectal cancers by Ion Torrent targeted sequencing and found frequent mutations in KRAS, TP53, APC, FBXW7, PI3KCA and, to a lesser extent, in BRAF, CTNNB1, ERBB2 and SMAD4. In addition, they identified associated multiple mutations, mainly involving KRAS and APC or KRAS and TP53. Harlé et al. [79] analyzed NRAS and KRAS in 188 consecutive mCRCs by using GS Junior technology. They identified nine uncommon mutational profiles and showed 4 undescribed nucleotide variants with aminoacid change, focusing the attention on the possibility to detect non-hotspot rare RAS mutations putatively able to impact the response to anti-EGFR. On the other hand, Taieb et al. [80] analyzed by real-time PCR 2559 stage III CRC patients treated with FOLFOX \pm cetuximab for hot-spot KRAS and BRAF V600E mutations. Nine-hundred height of them resulted positive. Of the remaining, 1054 were subjected to NGS (Ion Torrent, Ampliseq colon and lung) which allowed uncovering newly diagnosed K/NRAS and BRAF mutations in $227(21 \%)$ and $46(4.4 \%)$ patients, respectively. The authors described neither significant better trend of outcome in BRAF and RAS WT patients treated with FOLFOX plus cetuximab or detrimental effects in those RAS-mutant. However, they identified a clinically relevant 0.76 adjusted hazard ratio value evidenced for disease free survival (DFS) for cetuximab addiction in RAS and BRAF WT patients, suggesting a new randomized trial to test the efficacy of anti-EGFR in this setting and further pointing out the importance NGS analysis. In a very recent targeted exome-sequencing based work on 63 Iranian Shirazi patients, Ashktorab et al. [81] detected and further validated 51 variants in 12 genes by using two NGS platforms (Ion Torrent and Illumina). They showed higher mutation rate of MSH3, MSH6, APC and PI3KCA in Iranian patients, hypothesizing a major role of these genes in CRC and suggesting the adoption of specific informed genetic diagnosis protocol and tailored therapy in this population. Another study [82] confirmed detailed identification of mutations in $138 \mathrm{mCRC}$, identifying a novel KRAS mutation (KRAS ${ }^{\mathrm{R} 68 \mathrm{~S} 1}$ ) associated with an aggressive phenotype as well as rare RAS and MET amplification, BRAF and ARAF alterations, PTEN-PI3KCA-AKT 


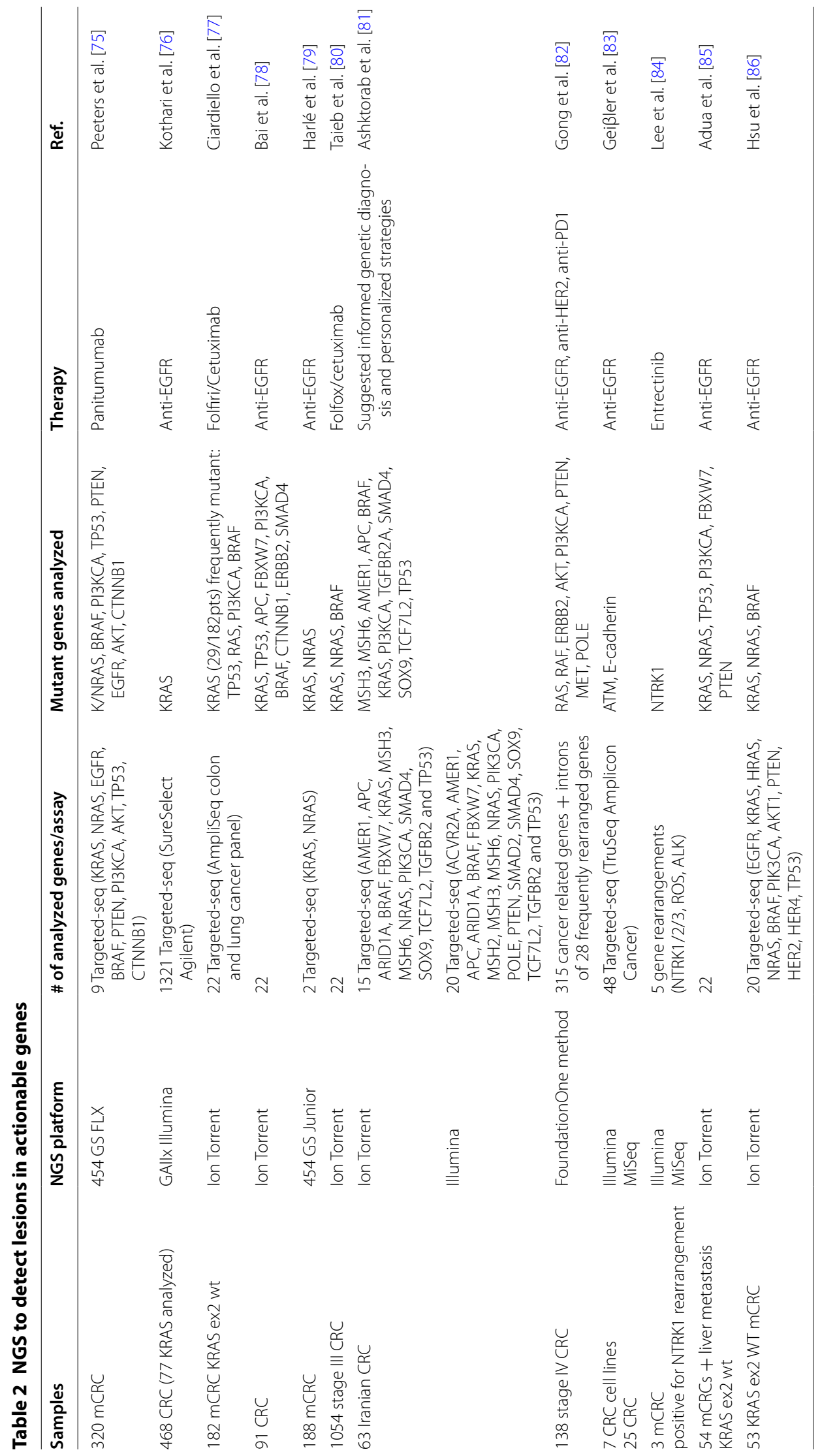


pathway mutations associated with poorer prognosis and, possibly, anti-EGFR resistance. The authors described three hypermutated tumors with MSI-H or POLE mutation and ERBB2 amplified tumors (5\% of cases) as well, which might benefit of anti-PD-1 or HER2-targeted therapy in absence of RAS/RAF mutations, respectively. Again, a recent study on both cell lines and tumor specimens [83] highlighted the importance of NGS in detecting genes potentially involved in the resistance to anti-EGFR therapy in KRAS wild-type patients. The authors analyzed the response of 7 CRC cell lines to cetuximab as well as primary tumors, liver and lung metastasis from 25 CRC patients treated with cetuximab or panitumumab, by evaluating NGS (Illumina) mutation profiles of 48 cancerrelated genes, EGFR and E-cadherin expression. Lack of response to anti-EGFR therapy was associated to ATM mutations and low E-cadherin expression, here described as novel supportive predictive markers. Lee et al. [84] analyzed NTRK1 rearrangements in $74 \mathrm{mCRC}$ and 66 gastric cancer (GC) patients. Two mCRC and one GC TrkAIHC positive patients were further analyzed by FISH and NGS, which highlighted TPM3-NTRK1 rearrangements. The use of entrectinib, a pan-TRK inhibitor, inhibited cell proliferation of patient-derived tumor cells (PDCs) with rearrangement by TrkA inactivation and downregulation of downstream pathways. In conclusion, this study focuses the attention on novel targeted drugs to be potentially used in presence of specific CRC gene lesions. A very recent work [85] analyzed the intra- and inter-tumor molecular heterogeneity between CRCs and synchronous liver metastasis. The authors studied the genotype of 22 genes (Ion Torrent, Ampliseq colon and lung panel), mainly involved in colorectal tumorigenesis, in primary tumor samples and liver metastasis from 7 KRAS-WT patients, both before and after chemotherapy associated to anti-EGFR. Results showed marked genotype differences by comparing pre- and post-therapy specimens, most probably attributable to tumor cells clones selected by the therapeutic treatment and, at the same time, able to affect the response to therapy. Several studies have further confirmed the NGS improvement in detecting mutation of BRAF, KRAS and EGFR-pathway genes. Fifty-three KRAS exon 2 WT mCRC patients, treated with cetuximab/irinotecan-based chemotherapy, were analyzed by using a panel of 10 genes related to EGFR pathway and NGS semi-conductor technology [86]. This study demonstrated that extensive analysis of EGFR pathway-related genes leads to the identification of variants with predictive value, which could help in individuating non-responders to targeted therapy. In particular, KRAS, NRAS and BRAF mutations are important factors in predicting response to cetuximab in KRAS exon 2 WT patients. Ma et al. [87] analyzed 822 cancers, including CRC, and compared results from Illumina (MiSeq or NextSeq) with those obtained by qRT-PCR based FDA-cleared testing kits, demonstrating that the latter can be now considered not sufficiently accurate. On the contrary, NGS is confirmed a powerful technology, due to the identification of a significant number of KRAS, BRAF, EGFR mutations which are missed by standard tests, but could have clinical relevance.

\section{Novel mutations or less frequently mutant genes}

Two studies, based on Sanger sequencing associated to bioinformatics approaches [88, 89], had already highlighted the importance of targeted multi-gene analysis in CRC. They shed light on the tumor heterogeneity and characterized a group of most commonly mutated as well as much larger number of genes less frequently mutated, but involved in several fundamental cell functions, such as transcriptional regulation, adhesion and invasion. As a result, 69 candidate genes with potential oncogenic driver mutations, including both cancer-related and previously uncharacterized genes, were identified, providing interesting insights potentially useful at the clinical level. Later, NGS-based studies provided interesting data (Table 3). Han et al. [90] analyzed 60 normal/tumor tissue pairs from colorectal adenocarcinoma patients by NGS (GAIIx Illumina) and considered 183 cancerrelated genes, known to predict response, therapeutically targetable, involved in major signaling pathways. The presence of 232 different somatic point mutations, 166 novels and 66 known, as well as copy number variations was highlighted. APC, TP53, KRAS were the most mutated genes and the ErbB pathway was described as the most affected, providing data about the usefulness of this method for clinical application. By using HiSeq whole-genome sequencing approach, Shanmugam et al. [91] analyzed a small number of refractory metastatic CRCs to possibly identify new therapies, and described mutations of several interesting genes. Beyond KRAS, APC, PI3KCA and TCF7L2 mutations, they discussed the significance of a lesion of INPPL1, a gene involved in PI3K/AKT signaling pathway. They identified the E567G aminoacid substitution in its SHIP2 protein product, a phosphatase which converts PIP3 to PIP2, negatively regulating PI3K/AKT signaling. The authors demonstrated that INPLL1 in vitro knock-down abolished cell growth, leaving to hypothesize that gene mutations might induce gain of function leading to cancer promotion and providing insights about its possible actionable role. In a work on 653 routine CRC, Malapelle et al. [92] analyzed a panel of 22 significant genes by using semiconductor-based technology. Besides the most frequently mutant genes, they described 12 additional genes carrying at least one mutations, highlighting potential actionable molecules 
Table 3 NGS to detect novel mutations or less frequently mutant genes

\begin{tabular}{|c|c|c|c|c|}
\hline Samples & NGS & \# analyzed genes & $\begin{array}{l}\text { Novel mutations/less frequently } \\
\text { mutant genes }\end{array}$ & Ref. \\
\hline 60 pairs normal/CRC & GAllx Illumina & 183 Targeted-seq (custom panel) & $\begin{array}{l}166 \text {-point mutations } \\
25 \text { indels }\end{array}$ & Han et al. [90] \\
\hline $4 \mathrm{mCRC}$ & $\begin{array}{l}\text { Illumina } \\
\text { HiSeq }\end{array}$ & $\begin{array}{l}\text { Whole genome (NEBNext DNA New } \\
\text { England) }\end{array}$ & INPPL1 p.E567G & Shanmugam et al. [91] \\
\hline $77 \mathrm{CRC}$ & Ion Torrent & $\begin{array}{l}22 \text { Targeted-seq (ampliseq colon and } \\
\text { lung) }\end{array}$ & $\begin{array}{l}\text { ALK p.L1196M/AKT1, STK11, ERBB2, } \\
\text { ERBB4, MAP2K1, NOTCH1 }\end{array}$ & Malapelle et al. [92] \\
\hline 14 HNPCC, 12 EC, 2 LS & $\begin{array}{l}\text { Illumina } \\
\text { HiSeq }\end{array}$ & 22 Targeted-seq (MMR custom panel) & POLD2, EXO1 & Talseth-Palmer et al. [93] \\
\hline $224 C R C$ & $\begin{array}{l}\text { Illumina } \\
\text { HiSeq }\end{array}$ & $\begin{array}{l}341 / 410 \text { cancer associated genes (Msk- } \\
\text { impact) }\end{array}$ & POLE p.P286R & Stadler et al. [94] \\
\hline $9643 \mathrm{mCRC}$ & Ion Torrent & $\begin{array}{l}50 \text { Targeted-seq (Ampliseq cancer } \\
\text { hotspot) }\end{array}$ & Non-V600 BRAF & Jones et al. [95] \\
\hline
\end{tabular}

in CRC. Interestingly, they identified for the first time in CRC the p.L1196M mutation on ALK, whose protein product induces high resistance to the RTK inhibitor Crizotinib in lung cancer. In addition, they described AKT1, STK11, ERBB2, ERBB4, MAP2K1, NOTCH1 infrequent mutations $(0.9-0.2 \%)$ as well. Talseth-Palmer et al. [93] analyzed by HiSeq a panel of 22 genes involved in MMR pathway in HNPCC and EC (endometrial cancer) patients and identified five exonic indels, 42 non-synonymous nucleotide substitutions and one intronic mutation. In CRC patients, one variant was classified likely pathogenic (MSH2, c.186_187 dup), two with uncertain relevance (EXO1, c.2212-1G > C; POLD2, c.203G > T) and 36 with unknown significance (in EXO1, LIG1, MLH1, MLH3, MSH3, MSH6, PMS1, PMS2, POLD1, RPA1 genes). The novel POLD2 c-203G > T variant was associated to the rare EXO1 c.2212-1G > C in a patient. Stadler et al. [94] analyzed (HiSeq) panels of 341 or, updated, 410 cancer associated genes in $224 \mathrm{CRCs}$ with available IHC staining for MMR. Among them, 193 specimens with less than 20 mutations were MMR-proficient, whereas 28 out of 31 showing more than 20 mutations were MMR-deficient (MMR-D). The remaining three samples evidenced more than 150 mutations and an ultramutator phenotype with somatic alterations at the level of POLE exonuclease domain (P286R) as well as additional mutations in more frequently mutant genes (KRAS, TP53, PI3KCA, KIT). The NGS analysis was cost effective, able to characterize both MMR and RAS/BRAF mutations and to provide a cutoff value for a "mutational load" which could be considered a very sensitive method to screen MMR-D cases. Finally, NGS data collected from more than $9600 \mathrm{mCRC}$ patients were analyzed to assess the presence and role of non-V600 BRAF mutations [95]. Non-V600 mutations were found in $2.2 \%$ (208) of all patients tested and accounted for $22 \%$ of all detected BRAF mutations. They seem to be correlated to a clinically distinct CRC subtype with better prognosis.

\section{NGS transcriptomics analysis}

Post-transcriptional events in CRC gained attention and interesting results have been collected by NGS (Table 4). Some scientists took advantage of NGS technology to sequence human CRC mRNAs to verify RNA changes, such as alternative cleavage and polyadenylation (APA), during CRC progression [96]. They analyzed APA in 15 CRC patients by Illumina platform and found many genes with progressive APA changes. Results were further validated in 50 patients, and five normal/tumor tissue pairs. Three genes (PPIE, DMKN, PDXK), with significant modifications by comparing normal mucosa/ adenoma/carcinoma, were proposed as potential biomarkers. In another study, Banky et al. [97] analyzed by GS Junior the alternative splicing pattern (ASP) of CD44, a gene associated with cancer and metastasis, in different human CRC cell lines. They provided a list of CD44 isoforms expressed by CRC, but absent in normal tissue. Furthermore, the CD44 isoforms' expression pattern remained constant both in CRC cells and primary and metastatic cancer xenografts. Moreover, they found high levels of CD44 v3 and v6 variants co-expression in tumor cells more prone to give raise to metastasis, suggesting a specific role of these splicing variants in CRC development and progression. Wu et al. [98] performed high throughput RNA-seq (Illumina) to compare CRC, adjacent non-tumor and distant normal tissues obtained from the same patient. The study revealed differentially expressed genes as well as alternative splicing, novel and fusion transcripts. Among the latter, the authors validated the tumor-restricted PTGFRN-NOTCH2. Due to the role of NOTHC2, considered a prognostic predictor linked to the "tumor differentiation status" in CRC, 

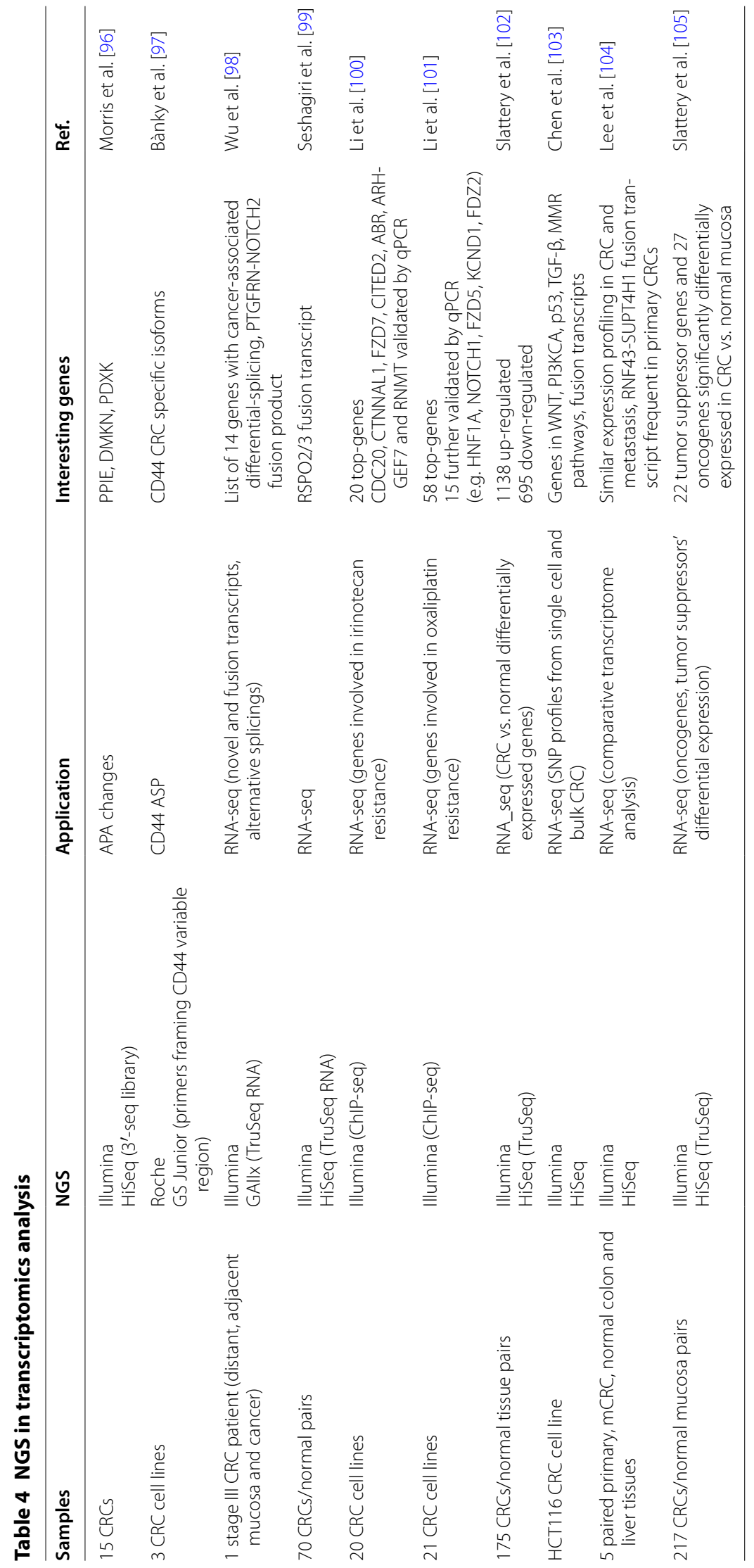
the authors hypothesized that this fusion transcript could have a dominant negative effect on normal cell development.

Wnt/APC pathway is altered in the most of CRC genomes, clearly demonstrating how this signaling is central in colorectal carcinogenesis. Its importance is further highlighted by the work of Seshagiri et al. [99] who analyzed more than 70 primary tumor pairs to characterize exome, transcriptome and copy number variation. They detected high numbers of mutations in many genes and identified 23 significantly mutated genes. By RNA-seq approach (Illumina), they discovered R-spondins (RSPO-2 and -3 ) fusion transcripts in $10 \%$ of primary CRCs. The R-spondins are secreted proteins able to potentiate canonical Wnt signaling. The presence of these aberrant transcripts seemed to mutually exclude APC and CTNNB1 mutations and, at the same way, to enhance WNT signaling, thus suggesting an alternative mechanism for WNT pathway aberrant activation. Li et al. [100] used 20 CRC cell lines to identify 20 top genes responsible for resistance/sensitivity to irinotecan and further validated 7 of them (CDC20, CTNNAL1, FZD7, CITED2, ABR, ARHGEF7 and RNMT) by qPCR in two resistant and sensitive CRC cell lines. Major differences, most probably indicating the involvement of them in the response to irinotecan, were detected in CTNNAL1, FZD7, CITED2 genes, overexpressed in the resistant cell line, and in ARHGEF7, overexpressed in the sensitive. A similar work [101], aimed to test the sensitivity to oxaliplatin, highlighted more than 50 top genes whose 15 were further validated: among them HNF1A, NOTCH1, FZD5 were negatively correlated to oxaliplatin resistance, and KCND1, FDZ2, positively correlated. The abovementioned works opened to novel biomarkers predicting the response to two of the most used chemotherapeutic drugs for CRC. Slattery et al. [102] analyzed 175 tumor/ normal tissue pairs from CRC patients and further processed almost 2000 differentially expressed genes to identify key pathways (cell signaling and growth). They concluded that having more dysregulated pathways is associated with a better prognosis, supporting this evidence with the observation that stage 1 patients have more dysregulated genes than those stage 4, probably due to the activation of more key events to arrest tumor progression. Chen et al. [103] performed SNP analysis by using RNA-seq data obtained by single-cell and bulk colorectal cancer cells. They assessed that single-cell RNA-seq is a powerful method to replicate the results of bulk analysis and, in addition, can reveal individual cell features not detectable in bulk-sample SNP analysis. After SNPs identification, GO (Gene Ontology) elaboration was performed and a list of cancer-related genes as well as pathway enrichment and fusion gene analysis were obtained. Mutations and fusion transcripts were identified at the level of genes involved in several key signaling pathways, including TGF- $\beta$, p53, PI3KCA, WNT and MMR. Lee et al. [104] showed by Illumina RNA-seq that gene expression patterns were highly similar in paired primary CRCs and liver metastasis, but, at the same time, identified fusion transcripts which were differentially expressed and could help to distinguish between primary tumor and metastasis. Among them, they described RNF43-SUPT4H1 in primary CRC, whose knock-down showed growth inhibitory effect. Slattery et al. [105] demonstrated the existence of co-regulatory networks involving tumor suppressor genes, oncogenes and miRNAs which might interact and play a role in regulating mechanisms of oncogenesis. To this aim, they analyzed 217 CRCs paired to normal mucosa and detected 22 differentially expressed tumor suppressor genes: 10 were up-regulated (FAM123B, RB1, TP53, RUNX1, MSH2, BRCA1, BRCA2, SOX9, NPM1, and RNF43), six downregulated (PAX5, IZKF1, GATA3, PRDM1, TET2, and CYLD), four were associated with MSI cancers (MLH1, PTCH1, and CEBPA down-regulated and MSH6 up-regulated) and two linked to MSS tumors (PHF6 and ASXL1 up-regulated). In addition, thirteen of those tumor suppressor genes were associated with 44 miRNAs. Among the oncogenes, 27 were dysregulated: 14 downregulated (KLF4, BCL2, SSETBP1, FGFR2, TSHR, MPL, KIT, PDGFRA, GNA11, GATA2, FGFR3, AR, CSF1R, and JAK3), 7 up-regulated (DNMT1, EZH2, PTPN11, SKP2, CCND1, MET, and MYC), 5 dysregulated in MSI (FLT3, CARD11, and ALK hypo-expressed, IDH2 and HRAS hyperexpressed), 1 up-regulated in MSS (CTNNB1). RNA-seq datasets available on the internet have been also used for in silico studies. Snezhkina et al. [106] analyzed Cancer Genome Atlas RNA-seq datasets obtained from CRC and normal tissue pairs. They found more than one thousand alternative mRNA isoforms involved in cell metabolism, identifying 7 genes whose alternative transcripts were differentially expressed in CRCs, even though their overall expression was not different. Eight differentially expressed isoforms encoded by OGDH, COL6A3, ICAM1, PHPT1, PPP2R5D, SLC29A1, and TRIB3, further validated by qRT-PCR, resulted up-regulated in $\mathrm{CRC}$, providing evidence about tumor specific alternative transcripts of genes involved in metabolism, which putatively play a role in CRC.

\section{Epigenetics analysis}

Next-gen technology has been also applied to epigenetics studies, principally focused on evaluating the methylation level or microRNAs expression in CRC. Some valuable profiling researches were conducted to sequence the methylome of CRC samples, being DNA 
methylation of several genes reported in CRC [107]. Hansen et al. performed a study by using Illumina platform (whole-genome bisulfite seq) proving the existence of blocks of hypomethylated regions encompassing a half of the genome [108]. They also reported the presence of certain cancer-specific differentially methylated regions characterized by high level of gene expression variability and demonstrated different degrees of methylation by comparing colon normal tissues, adenomas and carcinomas. Another study with the same platform confirmed these findings documenting that, in many CRC, genome regions of focal hypermethylation are concentrated into $\mathrm{CpG}$ islands and reside inside large hypomethylated blocks [109]. These sequences correspond to late replication and attachment to the nuclear lamina regions in human cell lines. Exciting possibilities offered by next-generation technology are clear also in the context of microRNA (miRNA) research, small noncoding RNA molecules able to post-transcriptionally regulate gene expression, involved in the control of many fundamental cell functions and considered as promising therapeutic targets and/or biomarkers [110, 111]. To this aim, a study focused on the differential expression of miRNAs between paired normal and tumor colon samples is explanatory. By using the Illumina GAIIx system, the authors described the discovery of 16 dysregulated miRNAs, previously undescribed in CRC. Among the most interesting hyper-expressed, they showed miR549 , located within the locus of KIAA1199 gene, already reported as strongly up-regulated in many colon carcinomas [112]. They suggested the possibility of using miR549 as early CRC surrogate biomarker, since it could be co-transcribed with KIAA gene. Later, Rohr et al. [113] analyzed, with Illumina GAII, miRNA and mRNA profiles from normal, primary tumor and metastasis tissues and identified 4 miRNAs (MiR-1, -129, -497, -215) as largely dysregulated in cancer. The authors performed a system-biology simulation aimed to analyze the effects of miRNA-1 as a putative therapeutic option, providing an in silico model for personalized cancer treatment. Recently, Koduru et al. [114] further confirmed the results of Rohr et al. by performing bioinformatics analysis on the same data. They identified 13 aberrantly expressed miRs in cancer and metastasis throughout the progression of the disease and evidenced very similar miRs' levels between primary tumors and metastasis. Neerincx et al. [115] analyzed miRNA expression profiling (HiSeq) in 220 fresh-frozen samples from paired primary colorectal cancers, metastases and non-tumor tissues. The study revealed approximately $222 \mathrm{miRs}$ able to differentiate primary tumors and metastasis from nontumor tissues. Among them, the most specific appeared to be miR-21 and miR-92a, already described as putative
CRC early diagnosis circulating biomarkers. On the contrary, only eight miRNAs, either already known or novel candidates (miR-320b, miR-320d, miR-3117, miR1246, miR-663b, chr 1-2552-5p, chr 8-20656-5p and chr 10-25333-3p), were differentially expressed in primary tumors compared to metastasis, indicating a comparable profiling which could be, however, of clinical utility to predict prognosis or response to therapies.

\section{Other NGS directions in CRC}

Recently, scientific literature was enriched with papers demonstrating that NGS can be suitable for the analysis of circulating tumor cells or cell-free DNA. This is a very interesting field with enormous potential to better understand the biological mechanisms at the base of cancer and to identify new diagnostic, prognostic or predictive biomarkers. With this regard, Heitzer et al. [116] demonstrated the possibility to use NGS technology (Illumina MiSeq) to identify mutations in single circulating tumor cells (CTCs) extracted from mCRC patients. A panel of 68 colorectal cancer-associated genes was used. Specifically, the mutational spectrum was compared in primary tumor, metastases and CTCs. The paper showed that some mutations in relevant genes (APC, KRAS, PI3KCA), previously found only in CTCs, could be uncovered at subclonal level also in the main tumor and in metastases of the same patient, thanks to the use of specific algorithms. NGS feasibility in this context is also confirmed by another recent work [117], where the authors show the possibility to detect with high sensitivity and specificity 568 mutations within six genes (EGFR, K/NRAS, BRAF, cKIT, PDGFRa) (SiRe panel) in cell-free DNA obtained from serum and blood samples from patients affected by mCRC, non-small cell lung cancer (NSCLC) and melanoma. The study was performed by semiconductor- based PGM. If confirmed, these evidences would allow to move towards less invasive tests to follow the progression of the disease and solve some practical issues, like tumor tissue unavailability. Although reiterating NGS feasibility, other studies pose some limits, particularly with respect to the overall clinical sensitivity [118]. Some of them suggest implementing NGS with mutant allele enrichment or use digital PCR to enhance reliability [119].

As above mentioned, the role of microbiome in CRC is a field of increasing interest. Encouraging data came from studies aiming to connect an altered gut microbiota with enhanced risk of CRC development. A research on fecal bacterial DNA in 94 healthy subjects and 47 CRC patients established that patients harboring tumors were characterized by reduced microbial community diversity [120]. In this case, CRC subjects seemed to have lower abundance of Clostridia and higher expression of pro-inflammatory genus such as Fusobacterium and 
Porphyromonas. Later, the same authors further demonstrated some inter-relations between fecal microbiota and metabolome, confirming the possible involvement of the latter genus in CRC [121]. NGS application resulted to be crucial for genetic sequencing of bacterial genomes in this context.

\section{Closing remarks: NGS pros and cons}

Next-generation sequencing technology can be considered as the future of high-throughput data analysis and genomic sequencing, providing a method to obtain high-throughput data with sensitivity and specificity. It offers the possibility to have massive parallel multigene sequencing in few hours, with significant time and cost reduction, by using very low amount of nucleic acids [122]. This is of great advantage for CRC and, in general, solid tumors clinical application, since the only available material is often derived from biopsied specimens. Of note, a work of Goswami et al. [123] described a list of pre-analytical factors to be considered to increase NGS success rate in this context, such as the quantity of DNA (it should be more than $10 \mathrm{ng}$ ), tumor cellularity, resection procedures and biopsied tumor dimensions. Concerning the cost, data from the NHGRI-funded genome sequencing groups indicate that a whole-exome sequence can be produced with approximately 1000 dollars [122]. The clear potential of these technologies is to enhance sequencing power, leading to more complete definition of the genomic landscape. This is important especially for the study of complicated diseases, such as cancer, because it permits to obtain a wider view of the genotype. Next-gen techniques can provide valuable data about mutational status, copy number variations, transcriptomics and epigenetics with the opportunity to combine current available single genetic tests into a unique test able to detect multiple variants. Great attention should be focused on the possibility to generate databases where the sequencing information of single patients can be stored, resulting accessible for future use by clinicians in terms of retrospective analysis and, possibly, therapeutic decisions. This is a fascinating scenario proposed in a review by Kamalakaran et al. [124]. But NGS technologies show also some challenging issues related to technical, clinical and regulatory/legal fields. Many efforts are currently in progress to adopt standardized procedures and initiatives for quality management. Standard procedures for sequencing workflows, at the pre-analytical, analytical and post-analytical level, standard procedures for sequence data handling, processing and storage have been proposed by companies, organizations and societies $[125,126]$. Recent published data by Ashktorab et al. [127] evidenced significant variability between two among the most common platforms, Illumina and Ion
Torrent, in their calling for nucleotide variants in CRC. Therefore, validation of NGS data by using additional sequencing methods, such as a second NGS platform or Sanger sequencing, is strongly suggested and can greatly improve the accuracy of the results obtained. Different NGS approaches have been conceived to shed light on colorectal carcinogenesis and to possibly isolate novel variants of clinical interest. For this purpose, the available tests progressed from hotspot in actionable gene, cancer-related and even more exhaustive gene panels. Free resources on the internet, such as the Genetic Testing Registry (GTR), are available to provide a central location for voluntary submission of genetic test information by providers (www.ncbi.nlm.nih.gov/gtr). With this regard, an important issue is about consensus panels to use for CRC NGS diagnostics. ESMO guidelines recommend testing exons 2, 3, 4 of KRAS and NRAS, essential for driving therapeutic decisions, and BRAF exon 15. In this review, we reported CRC NGS analyses directed to a variable number of cancer-related genes, ranging from more than 1300 to few genes, with common use of commercially available panels (Ampliseq, TruSeq). Among the genes included in the different panels used, KRAS, NRAS, BRAF, PI3KCA, EGFR are the most shared, due to their relevant role in CRC pathogenesis and treatment. Larger panels require longer time for procedure execution and higher cost, but, on the other hand, provide more extensive knowledge about mutational status, with the possibility to identify new genes of clinical utility for CRC management. In our opinion, KRAS, NRAS, BRAF and PI3KCA gene panel should at least be used. Also, issues related to the amount of NGS data should be addressed. These systems generate many $\mathrm{Gb}$ of data to analyze. It is challenging for bioinformaticians to organize and interpret these immense quantities of data. They need to use more powerful computers with advanced algorithms to perform analyses, with consequent problems in terms of both economic and human resources for small medical units or laboratories. Different bioinformatics tools are supplied with the NGS platforms, but additional measures are necessary to ameliorate good data generation and interpretation. Many physicians and researchers are actively promoting courses focused on updating the knowledge in the field. More genotypic and phenotypic data will be also needed to assess the impact of genomic variants in healthy and ill patients. Dong et al. [128] suggested a solution for these issues consisting in networking and partnership to have a solid panel of evidence-based results towards an improved understanding of the data. Furthermore, genetic sequencing raised regulatory concerns about its use in health structures. Regulation and approval of the procedure is essential as well as uniformity about methods and standards definition. 
Data storage and privacy should be standardized to avoid possible discriminations in the healthcare context: for example, in the field of health insurance. United States approved in 2008 the Genetic Information Nondiscrimination Act (GINA) to prevent these problems and legally regulate genetic testing data for public use.

\section{Conclusions}

Advances in technology made it possible to improve technical skills in nucleic acids sequencing. From the initial results of Sanger technique to the actual next-generation sequencing, a lot of work has been done trying to consider the "individual variability" to move to the "personalized medicine". Currently, NGS technology stands out as one of the most powerful and effective approach for fast DNA/RNA sequencing. In cancer research, many scientists are striving to exploit this technology at its best and some laboratories are starting to show exciting data, especially in the case of CRC. However, it should be noted that the amount of data in the field is still limited. Additional studies are required to obtain more significant reliability of this technology for clinical application. This means that, maybe, a proper optimization to discover the whole potential of these platforms could be achieved in some years from now. The concept of NGS use in clinical routine is challenging, since these tools produce good results in terms of detecting clinically relevant mutations, but often are not able to repeat these successful performances when wider regions of the genome are subjected to analysis. Specific improvements in quality control methods (i.e. the identification of correct quality parameters) could greatly help to overcome these problems. Additionally, the introduction of NGS technology as clinical tool will require for sure measures for process standardization, data handling and interpretation. Greater attention should be paid to the work of bioinformaticians and biostatisticians for the analyses of the massive quantity of data these systems will generate. Clinical challenges are principally based on obtaining accurate data which can be also easy to interpret, by taking into consideration critical issues related to somatic mutation detection in CRC and solid tumors, foremost the accuracy in identifying lesions with very low allelic frequencies. With this regard, innovative approaches for alignment, assembler and variant calling should be devised to augment the accuracy of the entire NGS workflow. Still today, bioinformatics approaches are agnostic about the disease under study and do not embed in their computation the knowledge specific to the disease or gene under analysis, as instead do the scientists in their evaluations. In this direction, a disruptive approach would be to devise new bioinformatics methods that are aware of the pathology and disease the scientists are looking for and add this knowledge while executing their analysis. In our opinion, this would considerably increase the accuracy of NGS results. At the same level, investments should be made for appropriate education and formation of clinicians about the interpretation of the clinical significance of the data obtained.

In conclusion, NGS technology surely represents a giant step forward in the direction toward personalized medicine against CRC, but further analyses are necessary to reach more complete results and higher level in our view of the big picture.

\section{Abbreviations \\ NGS: next generation sequencing; CRC: colorectal cancer; mCRC: metastatic colorectal cancer; FAP: familial adenomatous polyposis; MAP: MUTYH-asso- ciated polyposis; HNPCC: hereditary nonpolyposis colorectal cancer; MMR: mismatch repair; CIN: chromosomal instability; MSI: microsatellite instability; CIMP: CpG islands methylator phenotype; ROS: reactive oxygen species; NOS: nitrative oxygen species; VEGF: vascular endothelial growth factor; EGFR: epidermal growth factor receptor; FOLFOX: folinic acid, fluorouracil, oxaliplatin; FOLFIRI: folinic acid, fluorouracil, irinotecan; ZMW: zero mode-waveguide; EMT: epithelial-to-mesenchymal transition; 3'UTR: 3'untranslated region; CTC: circulating tumor cells.}

\section{Authors' contributions}

FDV wrote the manuscript, VM collected NGS CRC results, ADM and CC provided technical information about NGS technologies, DC, FZ CC and EA revised manuscript, AT conceived the topic and revised the manuscript. All authors read and approved the final manuscript.

\section{Author details}

${ }^{1}$ Division of Cancer Sciences, University of Southampton, Southampton, Hampshire SO16 6YD, UK. ${ }^{2}$ Department of Biotechnological and Applied Clinical Sciences, University of L'Aquila, L'Aquila 67100, Italy. ${ }^{3}$ Department of Medicine, Centre for Cell Signaling and Inflammation, Imperial College London, London W12 ONN, UK. ${ }^{4}$ Department of Molecular Medicine, La Sapienza University, Rome 00161, Italy.

\section{Acknowledgements}

Not applicable.

\section{Competing interests}

The authors declare that they have no competing interests.

Availability of data and materials

Not applicable.

\section{Consent for publication \\ Not applicable.}

\section{Ethics approval}

Not applicable.

\section{Funding}

This work was supported by MIUR-FIRB Grant No. RBAP10A9H9 to Edoardo Alesse. Fellowship to C. Compagnoni funded by the "Programma Operativo Nazionale Ricerca e Innovazione (PON R\&I 2014-2020, PhD Programme code DOT13SR6G7)".

\section{Publisher's Note}

Springer Nature remains neutral with regard to jurisdictional claims in published maps and institutional affiliations.

Received: 31 July 2017 Accepted: 27 November 2017

Published online: 08 December 2017 


\section{References}

1. Sanger F, Nicklen S, Coulson AR. DNA sequencing with chain-terminating inhibitors. Proc Natl Acad Sci USA. 1977;74:5463-7.

2. Meyerson M, Gabriel S, Getz G. Advances in understanding cancer genomes through second-generation sequencing. Nat Rev Genet. 2010;11:685-96.

3. Schadt EE, Turner S, Kasarskis A. A window into third-generation sequencing. Hum Mol Genet. 2010;19:R227-40.

4. Wei X, Ju X, Yi X, Zhu Q, Qu N, Liu T, et al. Identification of sequence variants in genetic disease using genes using targeted next generation sequencing. PLOS ONE. 2011:6:e29500.

5. Smith BC, McAndrew T, Chen Z, Harari A, Barris DM, Viswanathan S, et al. The cervical microbiome over 7 years and a comparison of methodologies for its characterization. PLoS ONE. 2012;7:e40425.

6. Ozretic L, Heukamp LC, Odenthal M, Buettner R. The role of molecular diagnostics in cancer diagnosis and treatment. Onkologie. 2012;35(Suppl 1):8-12.

7. Luo H, Sun C, Sun Y, Wu Q, Li Y, Song J, et al. Analysis of the transcriptome of Panax notoginseng root uncovers putative triterpene saponin biosynthetic genes and genetic markers. BMC Genom. 2011;12(Suppl 5):S5.

8. Kandoth C, McLellan MD, Vandin F, Ye K, Niu B, Lu C, et al. Mutational landscape and significance across 12 major cancer types. Nature. 2013:503:333-9.

9. Ding L, Ley TJ, Larson DE, Miller CA, Koboldt DC, Welch JS, et al. Clonal evolution in relapsed acute myeloid leukaemia revealed by wholegenome sequencing. Nature. 2012;481:506-10.

10. Li MM, Datto M, Duncavage EJ, Kulkarni S, Lindeman NI, Roy S, et al. Standards and guidelines for the interpretation and reporting of sequence variants in cancer: a joint consensus recommendation of the Association for Molecular Pathology, American Society of Clinical Oncology, and College of American Pathologists. J Mol Diagn. 2017:19(1):4-23.

11. Ferlay J, Soerjomataram I, Ervik M, Dikshit R, Eser S, Mathers C, Rebelo M, Parkin DM, Bray F. GLOBOCAN 2012 v.1.0 cancer incidence and mortality worldwide. Lyon: IARC CancerBase, International Agency for Research on Cancer, 2017, No. 11. http://globocan.iarc.fr. Accessed 15 Oct 2017.

12. Cirulli ET, Goldstein DB. Uncovering the roles of rare variants in common disease through whole genome sequencing. Nat Rev Genet. 2010;11:415-25

13. Guan YF, Li GR, Wang RJ, Yi YT, Yang L, Jiang D, et al. Application of next generations sequencing in clinical oncology to advance personalized treatment of cancer. Chin J Cancer. 2012;31(10):463-70.

14. Hodges E, Xuan Z, Balija V, Kramer M, Molla MN, Smith SW, et al. Genome-wide in situ exon capture for selective resequencing. Nat Genet. 2007;39(12):1522-7.

15. Sultan M, Schulz MH, Richard H, Magen A, Klingenhoff A, Scherf M, et al. A global view of gene activity and alternative splicing by deep sequencing of the human transcriptome. Science. 2008;321:956-60.

16. Ju YS, Kim Jl, Kim S, Hong D, Park H, Shin JY, et al. Extensive genomic and transcriptional diversity identified through massively parallel DNA and RNA sequencing of eighteen Korean individuals. Nat Genet. 2011;43:745-52

17. Fu Y, Sun Y, Li Y, Li J, Rao X, Chen C, et al. Differential genome-wide profiling of tandem $3^{\prime}$ UTRs among human breast cancer and normal cells by high-throughput sequencing. Genome Res. 2011;21:741-7.

18. Lee CY, Chiu YC, Wang LB, Kuo YL, Chuang EY, Lai LC, et al. Common applications of next-generation sequencing technologies in genomic research. Transl Cancer Res. 2013;2(1):33-45.

19. http://www.roche.com. 1 Oct 2017.

20. http://www.illumina.com. 1 Oct 2017.

21. http://www.thermofisher.com. 1 Oct 2017.

22. Hoppman-Chaney N, Peterson LM, Klee EW, Middha S, Courteau LK, Ferber MJ. Evaluation of oligonucleotide sequence capture arrays and comparison of next-generation sequencing platforms for use in molecular diagnostics. Clin Chem. 2010;56(8):1297-306.

23. Eid J, Fehr A, Gray J, Luong K, Lyle J, Otto G, et al. Real-time DNA sequencing from single polymerase molecules. Science. 2009;323(5910):133-8.

24. http://www.pacb.com. 1 Oct 2017
25. Jain M, Olsen HE, Paten B, Akeson M. The Oxford Nanopore MinION: delivery of nanopore sequencing to the genomics community. Genome Biol. 2016;17(1):239. Erratum in: Genome Biol. 2016;17(1):256.

26. Loose MW. The potential impact of nanopore sequencing on human genetics. Hum Mol Genet. 2017;26(R2):R202-7. https://doi.org/10.1093/ $\mathrm{hmg} / \mathrm{dd} \times 287$

27. https://nanoporetech.com. 1 Oct 2017.

28. http://www.qiagen.com, http://www.genereaderNGS.com. 1 Oct 2017.

29. Darwanto A, Hein AM, Strauss S, Kong Y, Sheridan A, Richards D, et al. Use of the QIAGEN GeneReader NGS system for detection of KRAS mutations, validated by the QIAGEN Therascreen PCR kit and alternative NGS platform. BMC Cancer. 2017;17(1):358. https://doi.org/10.1186/ s12885-017-3328-z

30. https://bionanogenomics.com. 1 Oct 2017.

31. Jaratlerdsiri W, Chan EKF, Petersen DC, Yang C, Croucher PI, Bornman MSR, Sheth P, Hayes VM. Next generation mapping reveals novel large genomic rearrangements in prostate cancer. Oncotarget. 2017:8(14):23588-602.

32. Goodwin S, McPherson JD, McCombie WR. Coming of age: ten years of next-generation sequencing technologies. Nat Rev Genet. 2016;17(6):333-51. https://doi.org/10.1038/nrg.2016.49.

33. Loman NJ, Misra RV, Dallman TJ, Constantinidou C, Gharbia SE, Wain J, et al. Performance comparison of benchtop high-throughput sequencing platforms. Nat Biotechnol. 2012;30(5):434-9.

34. Claesson MJ, Wang Q, O'Sullivan O, Greene-Diniz R, Cole JR, Ross RP, et al. Comparison of two next-generation sequencing technologies for resolving highly complex microbiota composition using tandem variable 16S rRNA gene regions. Nucleic Acids Res. 2010;38(22):e200.

35. Harismendy O, Ng PC, Strausberg RL, Wang X, Stockwell TB, Beeson KY, et al. Evaluation of next generation sequencing platforms for population targeted sequencing studies. Genome Biol. 2009;10(3):R32. https:// doi.org/10.1186/gb-2009-10-3-r32.

36. Bentley DR, Balasubramanian S, Swerdlow HP, Smith GP, Milton J, Brown $C G$, et al. Accurate whole human genome sequencing using reversible terminator chemistry. Nature. 2008;456(7218):53-9. https://doi. org/10.1038/nature07517.

37. Dohm JC, Lottaz C, Borodina T, Himmelbauer H. Substantial biases in ultra-short read data sets from high-throughput DNA sequencing. Nucleic Acids Res. 2008;36(16):e105. https://doi.org/10.1093/nar/gkn425.

38. Nakamura K, Oshima T, Morimoto T, Ikeda S, Yoshikawa H, et al. Sequence-specific error profile of Illumina sequencers. Nucleic Acids Res. 2011;39(13):e90. https://doi.org/10.1093/nar/gkr344.

39. Carneiro MO, Russ C, Ross MG, Gabriel SB, Nusbaum C, DePristo MA. Pacific biosciences sequencing technology for genotyping and variation discovery in human data. BMC Genom. 2012;5(13):375. https://doi. org/10.1186/1471-2164-13-375.

40. Koren S, Schatz MC, Walenz BP, Martin J, Howard JT, Ganapathy G, Wang $Z$, et al. Hybrid error correction and de novo assembly of single-molecule sequencing reads. Nat Biotechnol. 2012;30(7):693-700. https://doi. org/10.1038/nbt.2280

41. Frey KG, Herrera-Galeano JE, Redden CL, Luu TV, Servetas SL, Mateczun $\mathrm{AJ}$, et al. Comparison of three next-generation sequencing platforms for metagenomic sequencing and identification of pathogens in blood. BMC Genom. 2014;4(15):96. https://doi.org/10.1186/1471-2164-15-96.

42. Quail M, Smith ME, Coupland P, Otto TD, Harris SR, Connor TR, et al. A tale of three next generation sequencing platforms: comparison of Ion Torrent, pacific biosciences and illumina MiSeq sequencers. BMC Genom. 2012;13(1):341.

43. Liu L, Li Y, Li S, Hu N, He Y, Pong R, et al. Comparison of next-generation sequencing systems. J Biomed Biotechnol. 2012;2012:251364. https:// doi.org/10.1155/2012/251364

44. Russo G, Patrignani A, Poveda L, Hoehn F, Scholtka B, Schlapbach R, et al. Highly sensitive, non-invasive detection of colorectal cancer mutations using single molecule, third generation sequencing. Appl Transl Genom. 2015;16(7):32-9.

45. Misyura M, Zhang T, Sukhai MA, Thomas M, Garg S, Kamel-Reid S, et al. Comparison of next-generation sequencing panels and platforms for detection and verification of somatic tumor variants for clinical diagnostics. J Mol Diagn. 2016;18(6):842-50. 
46. Mu W, Lu HM, Chen J, Li S, Elliott AM. Sanger confirmation is required to achieve optimal sensitivity and specificity in next-generation sequencing panel testing. J Mol Diagn. 2016;18(6):923-32.

47. Hinrichs JW, van Blokland WT, Moons MJ, Radersma RD, Radersma-van Loon JH, de Voijs CM, et al. Comparison of next-generation sequencing and mutation-specific platforms in clinical practice. Am J Clin Pathol. 2015;143(4):573-8. https://doi.org/10.1309/AJCP40XETVYAMJPY.

48. Labianca R, Beretta GD, Kildani B, Milesi L, Merlin F, Mosconi S, et al. Colon cancer. Crit Rev Oncol Hematol. 2010;74(2):106-33.

49. Van Blarigan EL, Meyerhardt JA. Role of physical activity and diet after colorectal cancer diagnosis. J Clin Oncol. 2015;16:1825-34.

50. Yang B, Jacobs EJ, Gapstur SM, Stevens V, Campbell PT. Active smoking and mortality among colorectal cancer survivors: the Cancer Prevention Study II nutrition cohort. J Clin Oncol. 2015;8:885-93.

51. Sebastian S, Hernandez V, Myrelid P, Kariv R, Tsianos E, Toruner M, et al. Colorectal cancer in inflammatory bowel disease: results of the 3rd ECCO pathogenesis scientific workshop (I). J Crohns Colitis. 2014;1:5-18.

52. Kalla R, Ventham NT, Satsangi J, Arnott ID. Crohn's disease. BMJ. 2014;19(349):g6670.

53. Liu L, Lemmens VE, De Hingh IH, De Vries E, Roukema JA, Van Leerdam $\mathrm{ME}$, et al. Second primary cancers in subsites of colon and rectum in patients with previous colorectal cancer. Dis Colon Rectum. 2013;2:156-68.

54. Kastrinos F, Syngal S. Inherited colorectal cancer syndromes. Cancer J. 2011;6:405-15.

55. Fodde R. The APC gene in colorectal cancer. Eur I Cancer. 2002;38(7):867-71.

56. Vasen HF, Blanco I, Aktan-Collan K, Gopie JP, Alonso A, Aretz S, et al. Revised guidelines for the clinical management of Lynch syndrome (HNPCC): recommendations by a group of European experts. Gut. 2013;62(6):812-23.

57. Nielsen M, Lynch H, Infante E, Brand R. MUTYH-associated polyposis. In: Adam MP, Ardinger HH, Pagon RA, Wallace SE, Bean LJH, Mefford HC, Stephens K, Amemiya A, Ledbetter N, editors. GeneReviews. Seattle: University of Washington; 1993-2017.

58. Vogelstein B, Fearon ER, Hamilton SR, Kern SE, Preisinger AC, Leppert M, et al. Genetic alterations during colorectal tumor development. N Engl J Med. 1988;319:525-32.

59. Worthley DL, Leggett BA. Colorectal cancer: molecular features and clinical opportunities. Clin Biochem Rev. 2010;31(2):31-8.

60. Watanabe T, Kobunai T, Yamamoto Y, Matsuda K, Ishihara S, Nozawa $\mathrm{K}$, et al. Chromosomal instability (CIN) phenotype, CIN high or CIN low, predicts survival for colorectal cancer. J Clin Oncol. 2012;30(18):2256-64.

61. Boland CR, Goel A. Microsatellite instability in colorectal cancer. Gastroenterology. 2010;138(6):2073-2087.e3. https://doi.org/10.1053/j. gastro.2009.12.064.

62. Hinoue T, Weisenberger DJ, Lange CP, Shen H, Byun HM, Van Den Berg $D$, et al. Genome-scale analysis of aberrant DNA methylation in colorectal cancer. Genome Res. 2012;22(2):271-82.

63. Chan AO, Broaddus RR, Houlihan PS, Issa JP, Hamilton SR, Rashid A. $\mathrm{CpG}$ island methylation in aberrant crypt foci of the colorectum. Am J Pathol. 2002;160(5):1823-30

64. Vilar E, Gruber SB. Microsatellite instability in colorectal cancer-the stable evidence. Nat Rev Clin Oncol. 2010;7(3):153-62.

65. Winter SE, Lopez CA, Baumler AJ. The dynamics of gut associated microbial communities during inflammation. EMBO Rep. 2013;14:319-27.

66. Jobin C. Colorectal cancer: CRC - all about microbial products and barrier function? Nat Rev Gastroenterol Hepatol. 2012;9:694-6.

67. Arthur JC, Perez-Chanona E, Muhlbauer M, Tomkovich S, Uronis JM, Fan $\mathrm{TJ}$, et al. Intestinal inflammation targets cancer-inducing activity of the microbiota. Science. 2012;338:120-3.

68. Gagnière J, Raisch J, Veziant J, Barnich N, Bonnet R, Buc E, et al. Gut microbiota imbalance and colorectal cancer. World J Gastroenterol. 2016;22(2):501-18.

69. Gao R, Gao Z, Huang L, Qin H. Gut microbiota and colorectal cancer. Eur J Clin Microbiol Infect Dis. 2017;36(5):757-69.

70. Van Cutsem E, Cervantes A, Adam R, Sobrero A, Van Krieken JH, Aderka $D$, et al. ESMO consensus guidelines for the management of patients with metastatic colorectal cancer. Ann Oncol. 2016;27(8):1386-422.
71. Bruera G, Cannita K, Tessitore A, Russo A, Alesse E, Ficorella C, et al. The prevalent KRAS exon 2 c.35 G > A mutation in metastatic colorectal cancer patients: a biomarker of worse prognosis and potential benefit of bevacizumab-containing intensive regimens? Crit Rev Oncol Hematol. 2015;93(3):190-202.

72. Van Cutsem E, Köhne CH, Làng I, Folprecht G, Nowacki MP, Cascinu S, et al. Cetuximab plus irinotecan, fluorouracil, and leucovorin as first-line treatment for metastatic colorectal cancer: updated analysis of overall survival according to tumor KRAS and BRAF mutation status. J Clin Oncol. 2011;29:2011-20197.

73. Bokemeyer C, Bondarenko I, Hartmann JT, de Braud F, Schuch G, Zubel A, et al. Efficacy according to biomarker status of cetuximab plus FOLFOX-4 as first-line treatment for metastatic colorectal cancer: the OPUS study. Ann Oncol. 2011;22:1535-46.

74. Grada A, Weinbrecht K. Next-generation sequencing: methodology and application. J Invest Dermatol. 2013;133(8):e11. https://doi.org/10.1038/ jid.2013.248.

75. Peeters M, Oliner KS, Parker A, Siena S, Van Cutsem E, Huang J, et al. Massively parallel tumor multigene sequencing to evaluate response to panitumumab in a randomized phase III study of metastatic colorectal cancer. Clin Cancer Res. 2013;19(7):1902-12.

76. Kothari N, Schell MJ, Teer JK, Yeatman T, Shibata D, Kim R. Comparison of KRAS mutation analysis of colorectal cancer samples by standard testing and next-generation sequencing. J Clin Pathol. 2014;67(9):764-7.

77. Ciardiello F, Normanno N, Maiello E, Martinelli E, Troiani T, Pisconti S, et al. Clinical activity of FOLFIRI plus cetuximab according to extended gene mutation status by next-generation sequencing: findings from the CAPRI-GOIM trial. Ann Oncol. 2014;25(9):1756-61.

78. Bai J, Gao J, Mao Z, Wang J, Li J, Li W, et al. Genetic mutations in human rectal cancers detected by targeted sequencing. J Hum Genet. 2015;60(10):589-96.

79. Harlé A, Filhine-Tresarrieu P, Husson M, Boidot R, Rouyer M, Dubois C, Leroux A, Merlin JL. Rare RAS mutations in metastatic colorectal cancer detected during routine RAS genotyping using next generation sequencing. Target Oncol. 2016;11(3):363-70.

80. Taieb J, Balogoun R, Le Malicot K, Tabernero J, Mini E, Folprecht G, For PETACC8 Investigators, et al. Adjuvant FOLFOX \pm cetuximab in full RAS and BRAF wildtype stage III colon cancer patients. Ann Oncol. 2017;28(4):824-30.

81. Ashktorab H, Mokarram P, Azimi H, Olumi H, Varma S, Nickerson ML, et al. Targeted exome sequencing reveals distinct pathogenic variants in Iranians with colorectal cancer. Oncotarget. 2017;8(5):7852-66.

82. Gong J, Cho M, Sy M, Salgia R, Fakih M. Molecular profiling of metastatic colorectal tumors using next-generation sequencing: a single-institution experience. Oncotarget. 2017;8(26):42198-213.

83. Geißler AL, Geißler M, Kottmann D, Lutz L, Fichter CD, Fritsch R, Weddeling B, Makowiec F, Werner M, Lassmann S. ATM mutations and E-cadherin expression define sensitivity to EGFR-targeted therapy in colorectal cancer. Oncotarget. 2017;8(10):17164-90.

84. Lee SJ, Li GG, Kim ST, Hong ME, Jang J, Yoon N, et al. NTRK1 rearrangement in colorectal cancer patients: evidence for actionable target using patient-derived tumor cell line. Oncotarget. 2015;6(36):39028-35.

85. Adua D, Di Fabio F, Ercolani G, Fiorentino M, Gruppioni E, Altimari $A$, et al. Heterogeneity in the colorectal primary tumor and the synchronous resected liver metastases prior to and after treatment with an anti-EGFR monoclonal antibody. Mol Clin Oncol. 2017;7(1):113-20.

86. Hsu HC, Thiam TK, Lu YJ, Yeh CY, Tsai WS, You JF, et al. Mutations of KRAS/NRAS/BRAF predict cetuximab resistance in metastatic colorectal cancer patients. Oncotarget. 2016;7(16):22257-70. https://doi. org/10.18632/oncotarget.8076.

87. Ma W, Brodie S, Agersborg S, Funari VA, Albitar M. Significant improvement in detecting BRAF, KRAS, and EGFR mutations using next-generation sequencing as compared with FDA-cleared kits. Mol Diagn Ther. 2017. https://doi.org/10.1007/s40291-017-0290-z.

88. Sjöblom T, Jones S, Wood LD, Parsons DW, Lin J, Barber TD, et al. The consensus coding sequences of human breast and colorectal cancers. Science. 2006;314(5797):268-74.

89. Wood LD, Parsons DW, Jones S, Lin J, Sjöblom T, Leary RJ, et al. The genomic landscapes of human breast and colorectal cancers. Science. 2007;318(5853):1108-30. 
90. Han SW, Kim HP, Shin JY, Jeong EG, Lee WC, Lee KH, et al. Targeted sequencing of cancer-related genes in colorectal cancer using nextgeneration sequencing. PLoS ONE. 2013;8(5):e64271. https://doi. org/10.1371/journal.pone.0064271.

91. Shanmugam V, Ramanathan RK, Lavender NA, Sinari S, Chadha M, Liang WS, et al. Whole genome sequencing reveals potential targets for therapy in patients with refractory KRAS mutated metastatic colorectal cancer. BMC Med Genom. 2014;7:36.

92. Malapelle U, Pisapia P, Sgariglia R, Vigliar E, Biglietto M, Carlomagno C, et al. Less frequently mutated genes in colorectal cancer: evidences from next-generation sequencing of 653 routine cases. J Clin Pathol. 2016;69(9):767-71.

93. Talseth-Palmer BA, Bauer DC, Sjursen W, Evans TJ, McPhillips M, Proietto A, et al. Targeted next-generation sequencing of 22 mismatch repair genes identifies Lynch syndrome families. Cancer Med. 2016;5(5):929-41.

94. Stadler ZK, Battaglin F, Middha S, Hechtman JF, Tran C, Cercek A, et al. Reliable detection of mismatch repair deficiency in colorectal cancers using mutational load in next-generation sequencing panels. J Clin Oncol. 2016;34(18):2141-7.

95. Jones JC, Renfro LA, Al-Shamsi HO, Schrock AB, Rankin A, Zhang BY, et al. Non-V600 BRAF mutations define a clinically distinct molecular subtype of metastatic colorectal cancer. J Clin Oncol. 2017. https://doi. org/10.1200/JCO.2016.71.4394.

96. Morris AR, Bos A, Diosdado B, Rooijers K, Elkon R, Bolijn AS, et al. Alternative cleavage and polyadenylation during colorectal cancer development. Clin Cancer Res. 2012;18(19):5256-66.

97. Bánky B, Rásó-Barnett L, Barbai T, Tímár J, Becságh P, Rásó E. Characteristics of CD44 alternative splice pattern in the course of human colorectal adenocarcinoma progression. Mol Cancer. 2012;11(1):83.

98. Wu Y, Wang X, Wu F, Huang R, Xue F, Liang G, et al. Transcriptome profiling of the cancer, adjacent non-tumor and distant normal tissues from a colorectal cancer patient by deep sequencing. PLoS ONE. 2012;7(8):e41001. https://doi.org/10.1371/journal.pone.0041001.

99. Seshagiri S, Stawiski EW, Durinck S, Modrusan Z, Storm EE, Conboy CB, et al. Recurrent R-spondin fusions in colon cancer. Nature. 2012;488(7413):660-4.

100. Li XX, Zheng HT, Peng JJ, Huang LY, Shi DB, Liang L, et al. RNA-seq reveals determinants for irinotecan sensitivity/resistance in colorectal cancer cell lines. Int J Clin Exp Pathol. 2014;7(5):2729-36.

101. Li XX, Peng JJ, Liang L, Huang LY, Li DW, et al. RNA-seq identifies determinants of oxaliplatin sensitivity in colorectal cancer cell lines. Int J Clin Exp Pathol. 2014;7(7):3763-70.

102. Slattery ML, Herrick JS, Mullany LE, Gertz J, Wolff RK. Improved survival among colon cancer patients with increased differentially expressed pathways. BMC Med. 2015;13:75. https://doi.org/10.1186/s12916-015-0292-9.

103. Chen J, Zhou Q, Wang Y, Ning K. Single-cell SNP analyses and interpretations based on RNA-Seq data for colon cancer research. Sci Rep. 2016:6:34420. https://doi.org/10.1038/srep34420.

104. Lee JR, Kwon CH, Choi Y, Park HJ, Kim HS, Jo HJ, et al. Transcriptome analysis of paired primary colorectal carcinoma and liver metastases reveals fusion transcripts and similar gene expression profiles in primary carcinoma and liver metastases. BMC Cancer. 2016;16:539. https:// doi.org/10.1186/s12885-016-2596-3.

105. Slattery ML, Herrick JS, Mullany LE, Samowitz WS, Sevens JR, Sakoda L, et al. The co-regulatory networks of tumor suppressor genes, oncogenes, and miRNAs in colorectal cancer. Genes Chromosom Cancer. 2017. https://doi.org/10.1002/gcc.22481.

106. Snezhkina AV, Krasnov GS, Zaretsky AR, Zhavoronkov A, Nyushko KM, Moskalev AA, et al. Differential expression of alternatively spliced transcripts related to energy metabolism in colorectal cancer. BMC Genom. 2016;17(Suppl 14):1011. https://doi.org/10.1186/s12864-016-3351-5.

107. Ashktorab H, Rahi H, Wansley D, Varma S, Shokrani B, Lee E, et al. Toward a comprehensive and systematic methylome signature in colorectal cancers. Epigenetics. 2013;8(8):807-15

108. Hansen KD, Timp W, Bravo HC, Sabunciyan S, Langmead B, McDonald $\mathrm{OG}$, et al. Increased methylation variation in epigenetic domains across cancer types. Nat Genet. 2011:43(8):768-75.

109. Berman BP, Weisenberger DJ, Aman JF, Hinoue T, Ramjan Z, Liu $Y$, et al. Regions of focal DNA hypermethylation and long-range hypomethylation in colorectal cancer coincide with nuclear laminaassociated domains. Nat Genet. 2012;44(1):40-6.

110. Bartel DP. MicroRNAs: target recognition and regulatory functions. Cell. 2009;136(2):215-33.

111. Tessitore A, Cicciarelli G, Mastroiaco V, Vecchio FD, Capece D, Verzella D, et al. Therapeutic use of MicroRNAs in cancer. Anticancer Agents Med Chem. 2016;16(1):7-19.

112. Hamfjord J, Stangeland AM, Hughes T, Skrede ML, Tveit KM, Ikdahl T, et al. Differential expression of miRNAs in colorectal cancer: comparison of paired tumor tissue and adjacent normal mucosa using highthroughput sequencing. PLoS ONE. 2012;7(4):1-9.

113. Röhr C, Kerick M, Fischer A, Kühn A, Kashofer K, Timmermann B, et al. High-throughput miRNA and mRNA sequencing of paired colorectal normal, tumor and metastasis tissues and bioinformatic modeling of miRNA-1 therapeutic applications. PLoS ONE. 2013;8(7):e67461. https:// doi.org/10.1371/journal.pone.0067461.

114. Koduru SV, Tiwari AK, Hazard SW, Mahajan M, Ravnic DJ. Exploration of small RNA-seq data for small non-coding RNAs in Human Colorectal Cancer. J Genom. 2017;5:16-31.

115. Neerincx M, Sie DL, van de Wiel MA, van Grieken NC, Burggraaf JD, Dekker $\mathrm{H}$, et al. MiR expression profiles of paired primary colorectal cancer and metastases by next-generation sequencing. Oncogenesis. 2015;5(4):e170. https://doi.org/10.1038/oncsis.2015.29.

116. Heitzer E, Auer M, Gasch C, Pichler M, Ulz P, Hoffmann EM, et al. Complex tumor genomes inferred from single circulating tumor cells by array-CGH and next-generation sequencing. Cancer Res. 2013;73(10):2965-75

117. Malapelle U, Mayo-de-Las-Casas C, Rocco D, Garzon M, Pisapia P, Jordana-Ariza N, et al. Development of a gene panel for next-generation sequencing of clinically relevant mutations in cell-free DNA from cancer patients. Br J Cancer. 2017. https://doi.org/10.1038/bjc.2017.8.

118. Rachiglio AM, Esposito Abate R, Sacco A, Pasquale R, Fenizia F, Lambiase $M$, et al. Limits and potential of targeted sequencing analysis of liquid biopsy in patients with lung and coloncarcinoma. Oncotarget. 2016;7(41):66595-605.

119. Beije N, Helmijr JC, Weerts MJ, Beaufort CM, Wiggin M, Marziali A, et al. Somatic mutation detection using various targeted detection assays in paired samples of circulating tumor DNA, primary tumor and metastases from patients undergoing resection of colorectal liver metastases. Mol Oncol. 2016:S1574-7891(16):30110-7.

120. Ahn J, Sinha R, Pei Z, Dominianni C, Wu J, Shi J, et al. Human gut microbiome and risk for colorectal cancer. J Natl Cancer Inst. 2013;105(24):907-11.

121. Sinha R, Ahn J, Sampson JN, Shi J, Yu G, Xiong X, et al. Fecal microbiota, fecal metabolome, and colorectal cancer interrelations. PLoS ONE. 2016;11(3):e0152126. https://doi.org/10.1371/journal.pone.0152126.

122. http://www.genome.gov/sequencingcost. Accessed Sept 2017

123. Goswami RS, Luthra R, Singh RR, Patel KP, Routbort MJ, Aldape $K D$, et al. Identification of factors affecting the success of nextgeneration sequencing testing in solid tumors. Am J Clin Pathol. 2016;145(2):222-37.

124. Kamalakaran S, Varadan V, Janevski A, Banerjee N, Tuck D, McCombie WR, et al. Translating next generation sequencing to practice: opportunities and necessary steps. Mol Oncol. 2013;7(4):743-55.

125. Endrullat C, Glökler J, Franke P, Frohme M. Standardization and quality management in next-generation sequencing. Appl Transl Genom. 2016:10:2-9.

126. Matthijs G, Souche E, Alders M, Corveleyn A, Eck S, Feenstra I, et al Guidelines for diagnostic next-generation sequencing. Eur J Hum Genet. 2016;24(10):1515. https://doi.org/10.1038/ejhg.2016.63.

127. Ashktorab H, Azimi H, Nickerson M, Bass S, Varma S, Brim H. Targeted exome sequencing outcome variations of colorectal tumors within and across two sequencing platforms. Next Gener Seq Appl. 2016;3(1):123. https://doi.org/10.4172/2469-9853.1000123.

128. Dong L, Wang W, Li A, Kansal R, Chen Y, Chen H, et al. Clinical next generation sequencing for precision medicine in cancer. Curr Genom. 2015;16(4):253-63. 